\title{
Upregulation of Ganglioside GD2 Synthase (GD2S), as a New Putative Cancer Stem Cell Marker in Breast Carcinomas
}

\author{
Maryam Mansoori ${ }^{1,2}$, Alireza Mirzaei ${ }^{3}$, Isa Abdi Rad ${ }^{4}$, Rahim Mahmodlou ${ }^{5}$, Fatemeh Mansouri ${ }^{6}$, Leili Saeednejad \\ Zanjani $^{2}$, Zeynab Asadi - Lari ${ }^{7} * *$, Zahra Madjd ${ }^{1,2} *$ (D)
}

Received: 8 Jan 2019

Published: 6 Nov 2021

\section{Abstract}

Background: GD2 synthase (GD2S) is the key enzyme required for ganglioside GD2 synthesis. It is commonly expressed in normal tissues and various cancers. Ganglioside GD2 is identified as a breast cancer stem cells (BCSCs) marker that promotes tumorigenesis. As GD2S has been found to be a useful molecular marker in neuroblastoma and retinoblastoma tumors, we suggest that it can be considered as a suitable candidate for the detection of CSCs in breast cancer tissues.

Methods: Expression of GD2S was examined in 65 breast tumors compared to adjacent normal tissues, applying quantitative realtime PCR (qRT-PCR) and enzyme-linked immunosorbent assay (ELISA). The association between GD2S expression level and patients' clinical characteristics was also assessed.

Results: Our findings showed that GD2S mRNA expression was significantly higher in breast cancer tissues in comparison to normal adjacent tissue samples $(4.92$-fold change, $p<0.001)$ in advanced grades $(p<0.001)$ and stages $(p<0.001)$. It was also shown that GD2S protein expression was significantly higher in breast cancer tissues in comparison to normal adjacent tissues (4.86-fold change, $\mathrm{p}=0.010)$ in advanced grades $(\mathrm{p}=0.010)$, stages $(\mathrm{p}=0.005)$ and larger tumor size $(\mathrm{p}=0.002)$.

Conclusion: The current study showed that increased expression of GD2S in advanced breast cancer potentiates it as a promising tumor marker in these patients.

Keywords: Breast cancer, Cancer Stem Cells, ELISA, GD2 Synthase, qRT-PCR

Conflicts of Interest: None declared

Funding: This work was supported by a grant from Iran University of Medical Sciences (Grant \# 94-03-87-26452).

\section{*This work has been published under CC BY-NC-SA 1.0 license.}

Copyright $\odot$ Iran University of Medical Sciences

Cite this article as: Mansoori M, Mirzaei A, Abdi Rad I, Mahmodlou R, Mansouri F, Saeednejad Zanjani L, Asadi - Lari Z, Madjd Z. Upregulation of Ganglioside GD2 Synthase (GD2S), as a New Putative Cancer Stem Cell Marker in Breast Carcinomas. Med J Islam Repub Iran. 2021 (6 Nov);35:148. https://doi.org/10.47176/mjiri.35.148

\section{Introduction}

Breast cancer is the most common cancer among women worldwide, with 279,100 new cases expected in the United States in 2020 (1). The overall survival rate of

Corresponding author:Dr Zahra Madjd, majdjabari.z@iums.ac.ir

**Zeynab Asadi- Lari: Zeynab (21y) was a talented student at the University of Toronto who was tragically in the downed flight PS752, where her brilliant life and global perspectives were cut short on 8th January 2020.

1. Department of Molecular Medicine, Faculty of Advanced Technologies in Medicine, Iran University of Medical Sciences, Tehran, Iran

2. Oncopathology Research Center, Iran University of Medical Sciences (IUMS), Tehran, Iran

3. Bone and Joint Reconstruction Research Center, Shafa Orthopedic Hospital, Iran University of Medical Sciences, Tehran, Iran

4. Cellular and Molecular Research Center, Urmia University of Medical Sciences, Urmia, Iran

5. Department of Surgery, Imam Khomeini Hospital, Faculty of Medicine, Urmia University of Medical Sciences, Urmia, Iran

6. Department of Genetics and Immunology, Faculty of Medicine, Urmia University of Medical Sciences, Urmia, Iran

7. Department of Biology, Faculty of Science, University of Toronto, Toronto, Canada breast cancer has improved due to applying various tumor markers for population screening, diagno sis, staging, and prognosis. However, no specific breast tumor marker has

个What is "already known" in this topic:

Recent studies have supported the cancer stem cell (CSC) hypothesis, suggesting that many cancers are managed by cells possessing stem cell characters. Ganglioside GD2 has been introduced as a potential marker for breast CSCs. Synthesis of GD2 by GD2 synthase is considered to be critical for the synthesis of all complex gangliosides.

$\rightarrow$ What this article adds:

GD2 synthase mRNA and protein expression were significantly higher in breast cancer tissues in comparison to normal adjacent tissues in advanced grades and stages. We suggest that increased expression of GD2S in advanced breast cancer potentiates it as a promising tumor marker. 
been found so far (2).

The restriction of conventional therapies, like poor prognosis for patients at the advanced stages of cancer (3), usually includes systemic or local toxicity as well as drug resistance (4). This suggests that tumor bulk may involve a subpopulation of cells that are responsible for the start of tumor growth with the potential to metastasize and recur. Due to some resemblances between those cells and stem cells, the former has been named as CSCs (3). Also, recent studies have strongly supported the CSC hypothesis, suggesting that many cancers, including breast cancer, are driven by a subpopulation of cells displaying stem cell properties. These cells may mediate metastasis as well as contributing to treatment relapse due to their relative resistance to chemotherapy and radiation (5). According to the CSC theory, the CSC population may have diagnostic, prognostic, or clinical implications.

Some CSC markers may have a diagnostic potential, which is considered to be useful for early diagnosis (6). In addition, CSCs can also have prognostic potential that affects a patient's prognosis by promoting metastasis and recurrence (7). CSCs are involved in resistance and relapse after anticancer therapies. Thus, selective targeting and eradication of CSC are included in the most important therapeutic challenges, with clinical intervention potential (6). In earlier studies, various markers such as CD44 (8), EPCAM (9), and ALDH1 (10) have been introduced as potential markers of breast cancer stem cells (BCSC). However, attempts to explore a specific BCSC marker have not been successful yet. The clinical implication of BCSCs requires the identification of specific markers that have high relevance to the patients' clinical characteristics as well as outcome (11).

Recently, ganglioside GD2 has been introduced as a potential marker for breast CSCs (12). GD2 is introduced as a glycosphingolipid containing sialic acid, which is synthesized from GD3 by GD2 synthase. Moreover, it is also expressed on the cell surface of limited normal adult tissues, such as the central nervous system along with the surface of neuroblastomas and melanomas (13). $\beta 1,4-\mathrm{N}$ acetylgalactosaminyltransferase $\quad(\beta 1,4 \mathrm{GalNAc}-\mathrm{T} ; \quad \mathrm{EC}$ number: 2.4.1.92) is the key enzyme for the conversion of GM3, GD3, and lactosylceramide to GM2, GD2, and asialo-GM2 (GA2). Therefore, it is also called GM2/GD2 synthase (14).

Despite the potential relevance of GD2 in the diagnosis and prognosis of malignant tumors and therapeutics, the presence and role of GD2 require further investigations, especially in breast cancer (15).

Most previous studies conducted on breast cancer cell lines and BCSCs showed overexpression of GD2 synthase (GD2S) and an increased expression level in pc3-GD3s cells. Accordingly, GD2S was increased in BCSCs by 5.0fold. Notably, a study performed by Michael Andreeff on 12 breast cancer cell lines and 12 patient samples showed no elevation in GD2S, while they found GD3S in GD2 expression cells.

In BCSCs, GD2 and GD3 and their upstream glycosyltransferases, GD3 synthase (GD3S), and GD2 synthase (GD2S), can maintain stem cell phenotype (16). Also, it was found that small interfering RNAs against GD2S can efficiently reverse the proliferative phenotype $(17,18)$. The reduction of GD2 expression by GD2S knockdown reverted the CSC phenotype into a non-CSC phenotype (19).

We hypothesized that; GD2S might reflect the presence of CSCs in tissue samples of breast cancer patients. Therefore, we attempted to determine if levels of GD2S are elevated in tissue samples of BC patients compared to non-cancer patients. Consequently, performing this would provide support for GD2S as a CSC marker.

Previous studies showed controversial results. However, this is the first study with the largest sample size that compared GD2S expression between human breast cancer fresh tissue samples and normal adjacent tissues. Although several studies have been conducted on breast cancer cell lines, only one study was performed on 12 limited patient samples without making any comparison with normal tissues. Moreover, for the first time, in this study using statistical analysis, the potential relevance of this marker with the clinical characteristics of patients was evaluated. In the current study, we aimed to determine whether GD2S can be used as a proper tumor marker and also a potential CSC marker in breast cancer for future clinical applications.

\section{Methods}

\section{Patients}

A total of 65 patients with primary breast cancer, who underwent diagnostic surgery for breast cancer in the surgical department of Imam Khomeini hospital in Urmia, Iran, between 2015 and 2016, were enrolled in this study. After confirming malignancy, the normal adjacent tissues were also identified. Normal adjacent tissues of the same breast cancer patients were applied as the matched control. After confirming their diagnosis by the pathology report and providing written consent, the patients underwent mastectomy. Also, in this study, none of the patients underwent any treatment before the surgery. Accordingly, these patients comprised various subtypes of breast cancer including invasive ductal carcinoma, invasive lobular carcinoma, comedo type, micropapillary, metaplastic carcinoma, fibromatosis-like carcinoma, and ductal carcinoma in situ (20). Afterward, the archival medical records were retrieved to obtain the clinicopathological parameters including age, tumor type, histologic grade, tumor stage, lymphatic invasion, vascular invasion, and size of the tumor. Then, the tumors were staged and graded in terms of the TNM staging classification protocol (21) and BloomRichardson grading system (22), respectively.

Fresh tumor tissue samples were collected in RNA, and later RNA stabilization reagent was performed (Qiagen, Germany). For archival storage at $-80^{\circ} \mathrm{C}$ based on the Qiagen protocol, the tissues were incubated overnight in the reagent at $2^{\circ} \mathrm{C}$ to $8^{\circ} \mathrm{C}$, which were then removed from the reagent and were kept at $-80^{\circ} \mathrm{C}$. This study was approved by the ethics committee of Iran University of Medical Sciences (Ref No: IR.IUMS.rec.1394.26452). Moreover, the written informed consent was obtained from all the patients. 
RNA extraction, CDNA synthesis, and quantitative realtime PCR (qRT-PCR)

Total RNA was extracted from fresh tumor tissue samples, along with normal adjacent tissues, using RNX-plus solution, chloroform, isopropanol, and ethanol (all obtained from Afragen biotech, Iran). Total RNA (1-5 g) was reversely transcribed into cDNA in 20 L final volume using cDNA Synthesis Kit (Yekta Tajhiz Azma, YT4500, Iran). Subsequently, three biological replicates were processed for each sample. Quantitative real-time PCR (qRT-PCR) was then performed using iQ5 real-time PCR detection system (Bio-Rad, USA) for 1 cycle for 3 min at $95{ }^{\circ} \mathrm{C}$, followed by 40 cycles for $40 \mathrm{~s}$ at $95{ }^{\circ} \mathrm{C}$, and for $40 \mathrm{~s}$ at $60{ }^{\circ} \mathrm{C}$. Notably, SYBR Green qPCR master mix was also applied (Yekta Tajhiz Azma, YT2551, Iran). In this study, the primers were purchased from Pishgam Company (Pishgam, Iran). The sequence-specific primers were as follows: GD2 synthase F: GACAAGCCAGAGCGCGTTA and R: TACTTGAGACACGGCCAGGTT (23-25).

qRT-PCR data were analyzed using the comparative $\mathrm{Ct}$ method, which is a widely used method to present relative gene expression (also known as the $2^{-}{ }^{\mathrm{Ct}}$ method) (26). Afterward, relative expression levels of mRNA were normalized using the mRNA level of the reference gene, glyceraldehyde-3-phosphate dehydrogenase (GAPDH; primer: F: G GGGAGCCAAAAGGGTCATCATCT and R: GACGCCTGCTTCACCACC TTCTTG), which was applied as an endogenous control in each sample (27-29).

\section{Enzyme-linked immunosorbent assay (ELISA)}

A highly specific quantitative human B4GALNT1 sandwich ELISA kit (MBS109442, USA) was used to measure the protein expression of the GD2S in terms of the manufacturer's instructions. Subsequently, the absorb- ance was determined at $595 \mathrm{~nm}$ using an absorbance microplate reader (Awareness Technology, USA). Concentrations of cytokine are calculated based on a standard curve. The obtained data were represented as mean $\mathrm{ng} / \mathrm{mL}$ of GD2S \pm SD of three experiments.

\section{Statistical analysis}

Data were analyzed using the statistical software SPSS (version 20.0; IBM Corp, USA). In this study, we reported the categorical data by $\mathrm{N}(\%)$ and quantitative data as mean. A normality test was performed for checking the normal distribution of data. The mean level of GD2S expression was compared between fresh tumor tissue samples and adjacent normal tissues using Kruskal-Wallis and Mann-Whitney U tests. Pearson's $\chi 2$ was used to assess the association between gene and protein expression levels of GD2S as well as the clinicopathological parameters in the patients with breast cancer. A $\mathrm{P}$ value of $<0.05$ was considered statistically significant.

\section{Results}

Evaluation of GD2S MRNA expression level in fresh tumor tissues of breast cancer and their adjacent normal tissues using $q R T-P C R$

GD2S mRNA level in the tumors and their adjacent normal tissues was calculated by the expression of the gene of interest relative to the internal control in order to check if any differences may be caused by CSCs.

Based on our analysis, the relative mRNA level of GD2S was $6.43(\mathrm{SD}=0.8)$ and $1.35(\mathrm{SD}=0.1)$ in tumor tissues and in adjacent normal tissues, respectively (Fig. 1). Also, there was a 4.92-fold increase in the expression level of GD2S in tumor tissue samples of breast cancer patients compared to adjacent normal tissues. Accordingly, this difference was statistically significant $(\mathrm{p}<0.001)$.

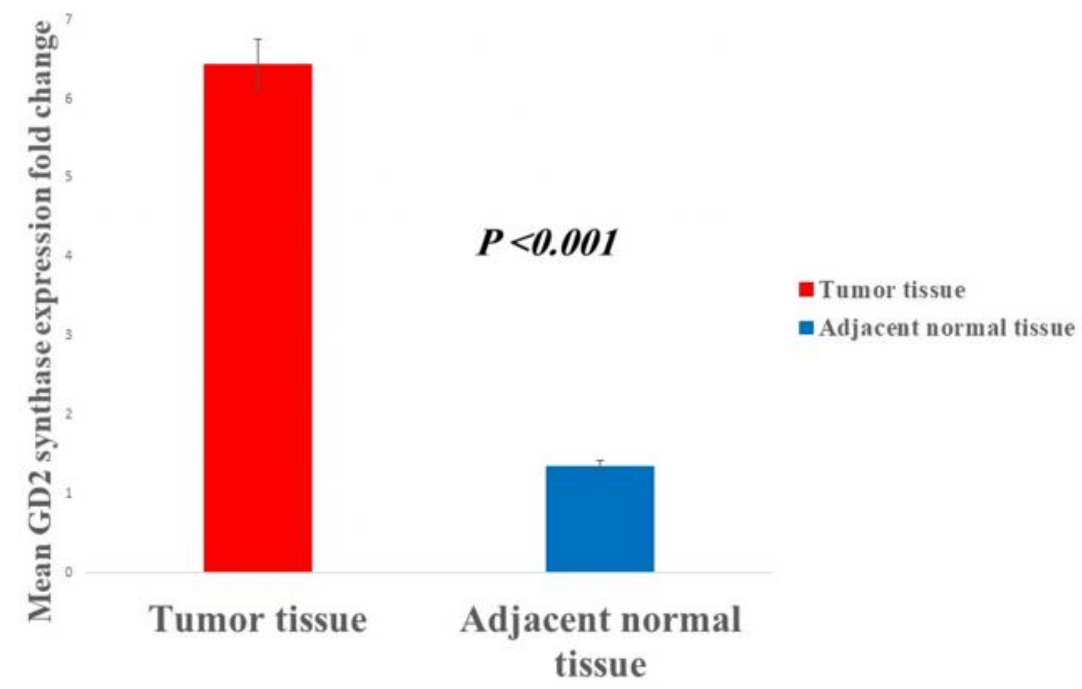

Fig. 1. GD2 synthase (GD2S) mRNA expression level in tumor tissue samples compared to normal adjacent tissues using qRT-PCR. The expression level of GD2S was significantly higher in tumoral tissue samples compared to adjacent normal tissues (4.92.-fold). Data are represented as mean $\pm \mathrm{SD}(\mathrm{n}=3$ each $)$. 


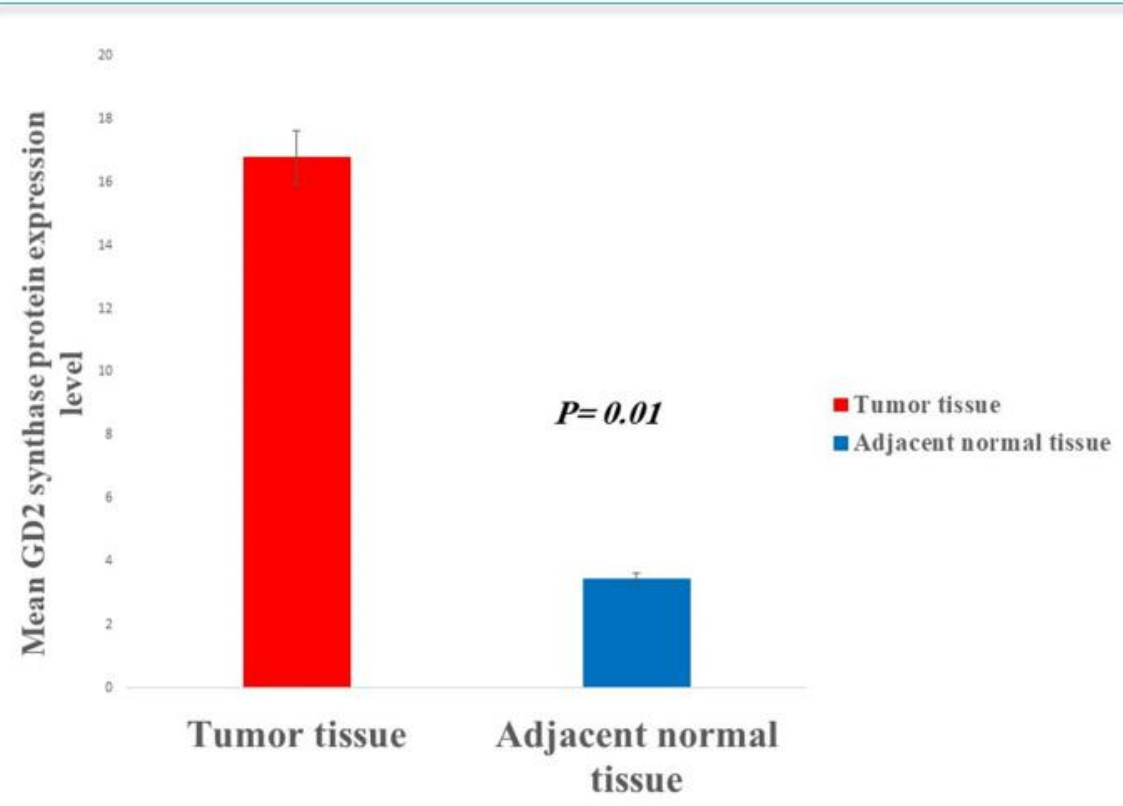

Fig. 2. GD2 synthase (GD2S) protein expression level in tumor tissue samples compared to normal adjacent tissues using ELISA. GD2S protein expression level was significantly higher in tumoral tissue samples compared to adjacent normal tissues (4.86-fold). Data are represented as mean $\pm \mathrm{SD}$ ( $\mathrm{n}=3$ each).

Evaluation of GD2S protein expression level in fresh tumor tissues of breast cancer and their adjacent normal tissues using ELISA

GD2S expression was compared in tumoral tissue samples instead of normal adjacent tissue samples to validate the protein expression of GD2S, which may be caused by the CSCs. A statistically significant increase was found in the expression of GD2S in tumoral tissue samples compared to normal adjacent tissue samples (4.86-fold, $\mathrm{p}=0.010)$ (Fig. 2).

The association between GD2S expression and clinicopathological characteristics of breast cancer tumor tissues

Patient characteristics: The sample population included a total of 65 patients. The mean age of the patients was 44 years old (ranged from 22 to 72 years old, $S D=10.59$ ), 36 $(55.4 \%)$ patients were younger than 44 years old, and 29 patients $(44.6 \%)$ were over 44 years old. In this study, tumor size (at the largest diameter) ranged from 0.2 to 7 $\mathrm{cm}$, which was then categorized into two groups, based on a mean tumor size of $3 \mathrm{~cm}$. The patient's clinicopathological features are summarized in Table 1.

mRNA expression: Pearson's chi-square test was used to evaluate the association between mRNA expression of GD2S and clinicopathologic characteristics of the patients with breast cancer. In this regard, the expression level of GD2S has significantly increased in higher grade tumors $(2 \& 3)(p<0.001)$ and at the advanced stages (III \& IV) compared to patients with stages I \& II breast cancer $(\mathrm{p}<0.001)$. The association among GD2S and the other clinicopathologic parameters including age, type of carcinoma, lymphatic invasion, and size of the tumor, was not statistically significant. The mean of relative expression was used as the cut-off value for the evaluation of the quantitative relative expression. These findings are summarized in Table 1.

Multiple linear regressions were used to predict the expression level of GD2S based on the clinicopathological characteristics, including grades and stages showing a significant correlation in a previous analysis. Moreover, a preliminary analysis was performed to ensure that there were no violations of the assumption of normality, linearity, and multi-co-linearity.

In this study, to test how the two independent variables (grade and stage) might affect the outcome (expression) and how they interact with each other to produce a unique response to the dependent variable (expression), a profile plot was prepared by SPSS. In addition, there was a significant stage (II, III \& IV) by grade ( $1 \& 2)$ interaction effect ( $\mathrm{p}=0.036$ ) on expression (Fig. 3).

Expression of GD2 protein: The expression of GD2S protein was associated with a higher grade $(\mathrm{p}=0.010)$, an advanced stage $(\mathrm{p}=0.005)$, and tumor size $(\mathrm{p}=0.002)$ in tumor tissue samples. Higher expression of GD2S was observed in $61.8 \%$ of the patients in grade 2 and $58.8 \%$ in the breast cancer patients at higher stages (III \& IV). Finally, higher expression of GD2S was observed in $73.5 \%$ of tumors with a size of $0.2-3 \mathrm{~cm}$ in breast cancer. Moreover, there was no significant association between GD2S protein expression and the other clinicopathological parameters of patients (Table 2). Preliminary analysis was performed to ensure that there were no violations of the assumption of normality, linearity, and multi-co-linearity.

In this study, in order to test how the two independent variables (grade and stage) might affect the outcome (protein level) and how they interact with each other to produce a unique response to the dependent variable (protein level), a profile plot was prepared by SPSS. In addition, 
M. Mansoori, et al.

Table 1. The association between GD2 synthase (GD2S) mRNA expression and clinicopathological parameters in fresh tumor tissues in patients with breast cancer (P-value; Pearson's $\chi 2$ test)

\begin{tabular}{|c|c|c|c|c|}
\hline \multirow{3}{*}{ Clinicopathological parameters } & \multicolumn{4}{|c|}{ GD2S expression } \\
\hline & \multicolumn{4}{|c|}{ mRNA expression } \\
\hline & Total samples n (\%) & Low & High & $\mathrm{P}$-value \\
\hline \multicolumn{5}{|l|}{ Mean age, years (22-72) } \\
\hline$\leq 44$ & $36(55.4)$ & $25(69.4)$ & $11(30.6)$ & \\
\hline$>44$ & $29(44.6)$ & $16(55.2)$ & $13(44.8)$ & 0.240 \\
\hline \multicolumn{5}{|l|}{ Tumor types } \\
\hline Invasive ductal carcinoma & $33(50.8)$ & $22(48.9)$ & $11(55)$ & \\
\hline Ductal carcinoma in situ & $9(13.8)$ & $7(15.6)$ & $2(10)$ & \\
\hline Comedo-type & $7(10.8)$ & $5(11.1)$ & $2(10)$ & 0.810 \\
\hline Metaplastic & $6(9.2)$ & $4(8.9)$ & $2(10)$ & \\
\hline Invasive lobular carcinoma & $6(9.2)$ & $4(8.9)$ & $2(10)$ & \\
\hline Fibromatosis-like & $3(4.6)$ & $2(4.4)$ & $1(5)$ & \\
\hline Micro papillary & $1(1.5)$ & $1(2.2)$ & $0(0)$ & \\
\hline \multicolumn{5}{|l|}{ Histological grade } \\
\hline 1 & $24(36.9)$ & $22(91.7)$ & $2(8.3)$ & \\
\hline 2 & $25(38.5)$ & $14(56.0)$ & $11(44.0)$ & $<0.001$ \\
\hline 3 & $16(24.6)$ & $5(31.2)$ & $11(68.8)$ & \\
\hline \multicolumn{5}{|l|}{ Tumor stage } \\
\hline I & $14(21.5)$ & $25(100.0)$ & $0(0.0)$ & \\
\hline II & $11(16.9)$ & $11(100.0)$ & $0(0.0)$ & $<0.001$ \\
\hline III \& IV & $40(61.5)$ & $5(17.2)$ & $24(82.8)$ & \\
\hline \multicolumn{5}{|l|}{ Lymphatic invasion } \\
\hline Yes & $36(55.4)$ & $23(79.3)$ & $6(20.7)$ & \\
\hline No & $29(44.6)$ & $18(50)$ & $18(50)$ & 0.300 \\
\hline \multicolumn{5}{|l|}{ Vascular invasion } \\
\hline Yes & $20(30.8)$ & $9(27.3)$ & $11(34.4)$ & \\
\hline No & $45(69.2)$ & $24(72.7)$ & $21(65.6)$ & 0.170 \\
\hline \multicolumn{5}{|l|}{ Tumor size $(\mathrm{cm})$} \\
\hline 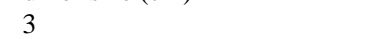 & $41(63.1)$ & $26(63.4)$ & $15(36.2)$ & \\
\hline$>3$ & $24(36.9)$ & $15(62.5)$ & $9(37.5)$ & 0.940 \\
\hline
\end{tabular}

$\mathrm{P}$ Value of $<0.05$ was considered statistically significant.

there was a significant stage (II, III \& IV) by grade $(1 \&$ 2) interaction effect $(\mathrm{p}<0.05)$ on expression (Fig. 4).

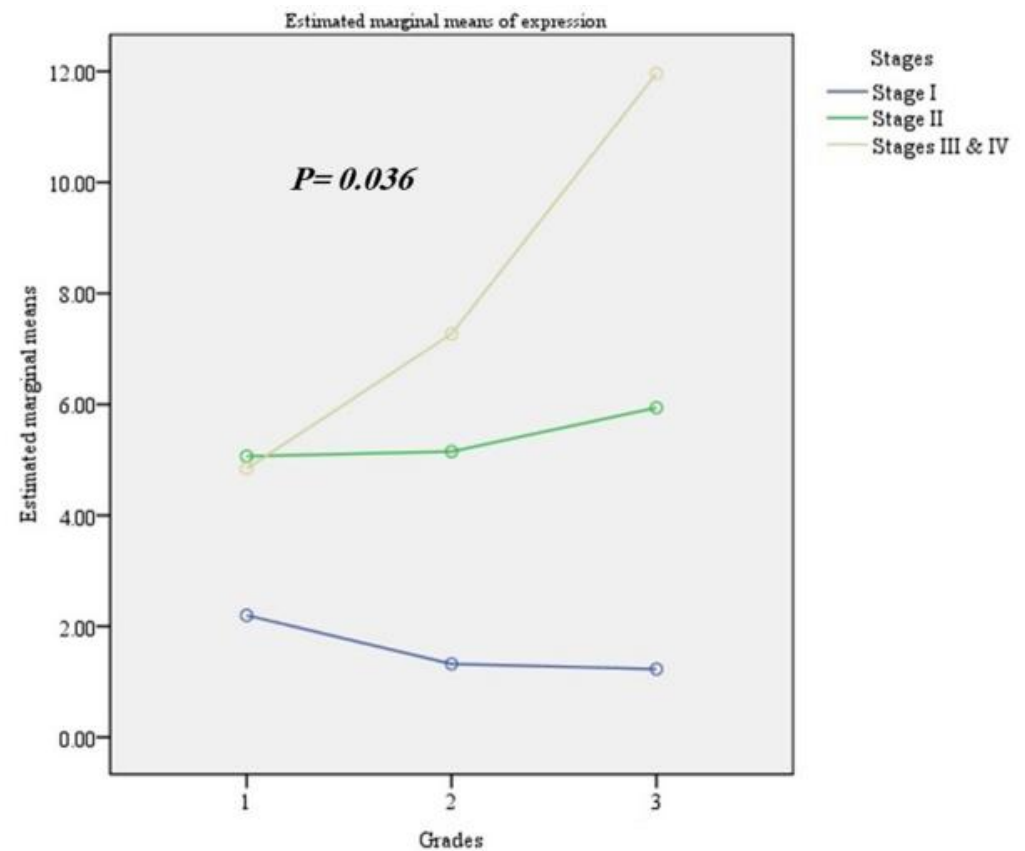

Discussion

Although incidence, mortality, and survival rates vary 4-fold in different regions worldwide, the incidence of

Fig. 3. Profile plot to evaluate interaction effects of grade and stage with GD2S mRNA expression. A significant tumor stage (II, III \& IV) by histological grade (1 \& 2) interaction effect $(\mathrm{P}=0.036)$ on expression was observed. 
Table 2. The association between GD2 synthase (GD2S) protein expression and clinicopathological parameters in fresh tumor tissues in patients with breast cancer (P-value; Pearson's $\chi 2$ test)

\begin{tabular}{|c|c|c|c|c|}
\hline \multirow{3}{*}{ Clinicopathological parameters } & \multicolumn{4}{|c|}{ GD2S expression } \\
\hline & \multirow{2}{*}{ Total samples n (\%) } & \multicolumn{2}{|c|}{ Protein expression } & \multirow[b]{2}{*}{$\mathrm{P}$-value } \\
\hline & & Low & High & \\
\hline \multicolumn{5}{|l|}{ Mean age, years (22-72) } \\
\hline$\leq 44$ & $36(55.4)$ & $15(48.4)$ & $15(44.1)$ & \\
\hline$>44$ & $29(44.6)$ & $16(51.6)$ & $19(55.9)$ & 0.460 \\
\hline \multicolumn{5}{|l|}{ Tumor types } \\
\hline Invasive ductal carcinoma & $33(50.8)$ & $1(3.0)$ & $32(97.0)$ & \\
\hline Ductal carcinoma in situ & $9(13.8)$ & $0(0.0)$ & $9(100.0)$ & \\
\hline Comedo-type & $7(10.8)$ & $0(0.0)$ & $7(100.0)$ & \\
\hline Metaplastic & $6(9.2)$ & $0(0.0)$ & $6(100.0)$ & \\
\hline Invasive lobular carcinoma & $6(9.2)$ & $0(0.0)$ & $6(100.0)$ & 0.400 \\
\hline Fibromatosis-like & $3(4.6)$ & $0(0.0)$ & $3(100.0)$ & \\
\hline Micro papillary & $1(1.5)$ & $0(0.0)$ & $1(100.0)$ & \\
\hline \multicolumn{5}{|l|}{ Histological grade } \\
\hline 1 & $24(36.9)$ & $11(35.5)$ & $3(8.8)$ & \\
\hline 2 & $25(38.5)$ & $9(29.0)$ & $21(61.8)$ & 0.010 \\
\hline 3 & $16(24.6)$ & $11(35.5)$ & $10(29.4)$ & \\
\hline \multicolumn{5}{|l|}{ Tumor stage } \\
\hline I & $14(21.5)$ & $11(35.5)$ & $0(0.0)$ & \\
\hline II & $11(16.9)$ & 7 (22.6) & $14(41.2)$ & 0.005 \\
\hline III \& IV & $40(61.5)$ & $13(41.9)$ & $20(58.8)$ & \\
\hline \multicolumn{5}{|l|}{ Lymphatic invasion } \\
\hline Yes & $36(55.4)$ & $18(58.1)$ & $18(52.9)$ & \\
\hline No & $29(44.6)$ & $13(41.9)$ & $16(47.1)$ & 0.430 \\
\hline \multicolumn{5}{|l|}{ Vascular invasion } \\
\hline Yes & $20(30.8)$ & $9(29.0)$ & $11(32.4)$ & \\
\hline No & $45(69.2)$ & $22(71.0)$ & $23(67.6)$ & 0.490 \\
\hline \multicolumn{5}{|l|}{ Tumor size $(\mathrm{cm})$} \\
\hline $\mathfrak{3}$ & $41(63.1)$ & $11(35.5)$ & $25(73.5)$ & \\
\hline$>3$ & $24(36.9)$ & $20(64.5)$ & $9(26.5)$ & 0.002 \\
\hline
\end{tabular}

$\mathrm{P}$ Value of $<0.05$ was considered statistically significant.

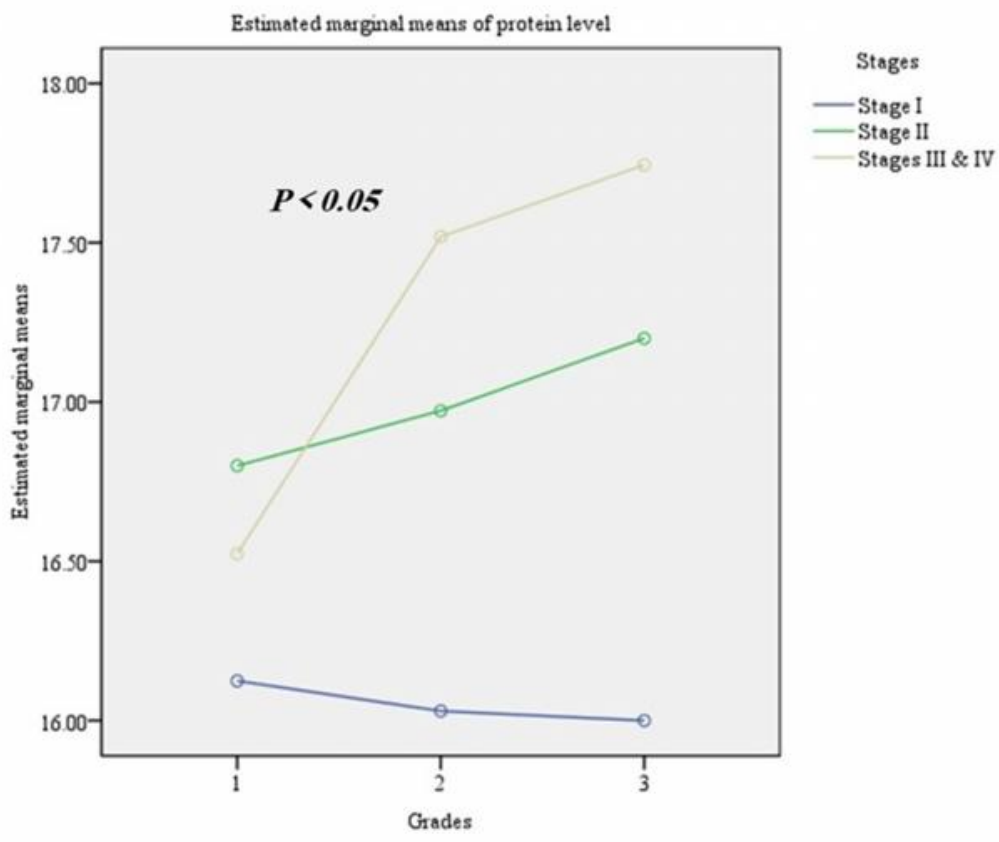

Fig. 4. Profile plot to evaluate interaction effects of grade and stage with GD2S protein expression. A significant tumor stage (II, III \& IV) by histological grade (1 \& 2) interaction effect $(\mathrm{p}<0.05)$ on expression was observed.

breast cancer is increasing all around the world, and mortality is also increasing in those regions that lack early detection programs (30).

Several markers are used as tumor markers in patients with breast cancer. However, the available markers have no sensitivity for early disease, and they all lack specificity, so they are not of value in early detecting breast cancer (31). 
CSC theory not only elucidates the issue of tumor's initiation, development, and ability to metastasis and reoccur, but it also indicates the inefficacy of conventional cancer therapy (3).

BCSCs were firstly determined using CD24 -/low $/ \mathrm{CD} 44^{+}$ population, showing the intensified ability to commence tumor growth when xenografted into immunocompromised mice (8). Several other markers were subsequently determined such as CD133, ALDH1, alpha-6 integrin/CD49f, SOX2, CK5, beta-1 integrin/CD29, ER-. Accordingly, cells containing these phenotypes are also found in cell lines of breast cancer and may impress cells with the expanded ability for tumor initiation (11).

Despite considerable progress in understanding CSC surface molecules, there still exist many issues to be addressed. Moreover, it should be noted that none of the CSC markers are specific up to now, and all CSCs do not express the markers. However, some non-CSCs may express the markers. Although the markers can be applied for the determination of CSC-rich subpopulations, all the CSCs may not be isolated by them precisely (32). One of the superiority of GD2 comparing other tumor-associated gangliosides is the fact that GD2 is highly expressed in tumor cells, while it is not expressed or exhibits a very low-level expression in normal cells (33). Notably, no staining or very low level of expression of GD2 in stroma cells has been reported in previous studies. In this regard, Gangliosides GD2 and GD3 are widely distributed in the central nervous system (CNS) and at lower levels in the stroma of most organs (27). GD2 expression in normal fetal and adult tissues was primarily restricted to the central nervous system, peripheral nerves, and skin melanocytes; however, a low level of GD2 expression was described in the stromal component of some normal tissues including mesenchymal stromal cells (MSCs), and white pulp of the spleen (28). In a very recent study by Massimo Dominici et al., only a few GD2 positive cells were observed within the negative stroma. Afterward, the specificity of GD2 staining on breast cancer cells was confirmed by no staining apparent in stroma and normal glandular ducts (29).

Synthesis of GD2 by GD2S enzyme is considered to be critical for the synthesis of all complex gangliosides enriched in the nervous system of vertebrates. Also, substantial evidence for the role of complex gangliosides has been obtained following the cloning of cDNAs encoding GM2/GD2S using an expression-cloning approach (14).

The key enzyme for GD2 synthesis (GD2S) in mRNA level was evaluated as a molecular marker to identify the neuroblastoma cells with metastatic behavior in bone marrow (34). However, to date, no study has been conducted either on clinical specimens or on the relevance of GD2S expression with the clinicopathological characteristics.

Therefore, this study was conducted to evaluate GD2S expression in fresh tumor tissues of breast cancer and the normal adjacent tissues. Also, in this study, for the first time, the relevance of GD2S level with the patients' clinical characteristics was assessed. The current study found that GD2S expression was higher in tumor tissues compared to the normal adjacent tissues, which was associated with the grade and stage of cancer.

Sen-itiroh Hakomori et al. (35) conducted a study on breast cancer cell lines. In their study, it was indicated that among various glycosyltransferases, mRNA levels have increased in CSCs for ST3GAL5, B4GALNT1, ST8SIA1, and ST3GAL2, which may clarify the enhanced expressions of GD2, GD3, GM2, and GD1a in CSCs. Moreover, knockdown of B4GALNT1 and ST8SIA1 significantly decreased the expressions of GD2 and GD3 and caused a phenotype change from CSC to a non-CSC that could be found by the reduced mammosphere formation and cell motility.

In a recent study conducted by Cheorl-Ho Kim (36), it was also found that the intracellular ganglioside GD3 is transformed to GD2 type (more specifically to Galactose$\beta 1$, 4-GalNAc transferase) possibly through intracellular endogenous GD2S. In this regard, although the GD2S mRNA was expressed in both pc3-GD3s (GD3S synthase (GD3S) over-expressing cells) and pc3 (pcDNA3) cells, and enhanced expression was seen in the pc3-GD3s cells. Accordingly, results proposed that the GD3S-transfected cells accumulated GD2 ganglioside formed from GD3 precursors. In a study on 12 different breast cancer cell lines and samples, Michael Andreeff et al. (12) showed that GD3S is highly expressed in GD2+ and $\mathrm{CD} 44^{\text {high }} / \mathrm{CD} 24^{\text {low }}$ cells, not GD2S.

Our study was conducted on fresh tumors and adjacent normal tissues in the patients with breast cancer, while previous studies focused on cell lines and a limited number of the patients' samples $(n=12)$. Moreover, in the present study, for the first time, we compared GD2S expression with the clinicopathological characteristics of breast cancer patients in both levels of gene and protein expressions.

The majority of the previous studies $(35,36)$ on breast cancer cell lines showed overexpression of GD2S. Also, an increased expression level was observed in the pc3GD3s cells. Moreover, in other studies, GD2S expression increased by 5.0- fold in BCSCs. However, in the abovementioned study performed by Michael Andreeff, GD3S in GD2 expressing cells showed overexpression.

In addition, we found an elevated level of GD2S in tumor tissues compared to normal tissues. In our previous study, 168 tissue samples embedded in formalin-fixed paraffin were immune-stained for GD2 by immunohistochemistry. Higher GD2 expression was found in the patients with breast cancer with advanced histological grade, presence of lymph node invasion, a larger size of tumors, and older ages. Although overexpression of GD2 was observed in tumoral cells in comparison to a very low level of expression in normal adjacent tissues, no expression of GD2 was observed in stromal cells (37).

Also, previous studies showed the same trend as they only evaluated cell lines of breast cancer, but not the tissue samples. Correspondingly, Andreef showed no increase in GD2S level in GD2 positive cells but GD3S overexpression. However, their study had a small sample size of 12 cell lines and 12 patient samples, without making any comparison to normal tissue or to the patient's clinicopathological characteristics. The only similar study 
that assessed the relevance of GD2 with 63 samples for IHC showed no relationship with the important clinicopathological parameters. However, in this study, we found a significant correlation between GD2S and the grade and stage of the disease.

For further assessment, it is recommended to examine the relationship between GD2S and GD2 overexpression with larger sample size. Also, it is suggested to study GD2S in cellular levels.

\section{Conclusion}

According to our results, the GD2S expression level was higher in breast cancer tumor tissues compared to the normal adjacent tissues. This elevation was found that GD2S expression may be associated with the grade and stage of breast cancer. These findings may suggest GD2S as a promising tumor marker of breast cancer for clinical application.

\section{Acknowledgments}

The authors would like to thank the staff of Oncopathology Research Center, Iran University of Medical Sciences, as well as Cellular and Molecular Biology Research Centre of Urmia University of Medical Sciences and Department of Pathology Imam Khomeini Hospital of Urmia.

\section{Conflict of Interests}

The authors declare that they have no competing interests.

\section{List of abbreviations}

GD2: ganglioside GD2; CSC: Cancer Stem Cell; GD2S: GD2 synthase; PCR: Polymerase Chain Reaction; BC: Breast Cancer; BCSC: Breast Cancer Stem Cell; GD3S: GD3 synthase; cDNA: complementary DNA

\section{Ethics approval and consent to participate}

The study was approved by Iran University of Medical Sciences Human Research Ethics Committee in Iran (Ref No: IR.IUMS.rec.1394.26452). All the procedures were performed in accordance with the 1964 Helsinki Declaration and its later amendments. Informed consent was obtained from all individual participants included in the study.

\section{References}

1. Siegel RL, Miller KD, Jemal A. Cancer Statistics, 2020. CA CANCER J CLIN. 2020;70(7).

2. Kumar K, Jain P, Sinha A, Singh KK, Sharma HP. Clinical Significance of Tumour Markers. AJPCT. 2014;2(8):1005-15.

3. Gil J, Stembalska A, Pesz KA, Sasiadek MM. Cancer stem cells: the theory and perspectives in cancer therapy. J Appl Genet. 2008;49(2):193-9.

4.Han L, Shi S, Gong T, Zhang Zh, Sun X. Cancer stemcells:therapeuticimplicationsandperspectives in cancertherapy. Acta Pharm Sin B. 2013;3(2):65-75.

5. Liu S, Cong Y, Wang D, Sun Y, Deng L, Liu Y, et al. Breast cancer stem cells transition between epithelial and mesenchymal states reflective of their normal counterparts. Stem Cell Rep. 2014;2(1):7891.

6. Ji J, Wang XW. Clinical implications of cancer stem cell biology in hepatocellular carcinoma. Semin Oncol. 2012;39(4):461-72.
7. Romano M, De Francesco F, Pirozzi G, Gringeri E, Boetto R, Di Domenico M, et al. Expression of cancer stem cell biomarkers as a tool for a correct therapeutic approach to hepatocellular carcinoma. Oncoscience. 2015;2(5):443-56.

8. Al-Hajj M, Wicha MS, Benito-Hernandez A, Morrison SJ, Clarke MF. Prospective identification of tumorigenic breast cancer cells. Proc Natl Acad Sci U S A. 2003;100(7):3983-8.

9. Wang N, Shi L, Li H, Hu Y, Du W, Liu W, et al. Detection of circulating tumor cells and tumor stem cells in patients with breast cancer by using flow cytometry: a valuable tool for diagnosis and prognosis evaluation. Tumour Biol. 2012;33(2):561-9.

10. Charafe-Jauffret E, Ginestier C, Iovino F, Wicinski J, Cervera N, Finetti $\mathrm{P}$, et al. Breast cancer cell lines contain functional cancer stem cells with metastatic capacity and a distinct molecular signature. Cancer Res. 2009;69(4):1302-13.

11. Liu Y, Nenutil R, Appleyard MV, Murray K, Boylan M, Thompson $\mathrm{AM}$, et al. Lack of correlation of stem cell markers in breast cancer stem cells. Br J Cancer. 2014;110(8):2063-71.

12. Battula VL, Shi Y, Evans KW, Wang RY, Spaeth EL, Jacamo RO, et al. Ganglioside GD2 identifies breast cancer stem cells and promotes tumorigenesis. J Clin Invest. 2012;122(6):2066-78.

13. Roth M, Linkowski M, Tarim J, Piperdi S, Sowers R, Geller D, et al. Ganglioside GD2 as a Therapeutic Target for Antibody-Mediated Therapy in Patients With Osteosarcoma. Cancer. 2014;120(548-54).

14. Koichi Furukawa, Kogo Takamiya, Furukawa K. h1,4-Nacetylgalactosaminyltransferase-GM2/GD2 synthase: a key enzyme to control the synthesis of brain-enriched complex gangliosides. Biochim Biophysic Acta. 2002;1573:356-62.

15. Orsi G, Barbolini M, Ficarra G, Tazzioli G, Manni P, Petrachi T, et al. GD2 expression in breast cancer. Oncotarget. 2017;8(19):31592600 .

16. Liang YJ, Ding Y, Levery SB, Lobaton M, Handa K, Hakomori SI. Differential expression profiles of glycosphingolipids in human breast cancer stem cells vs. cancer non-stem cells. Proc Natl Acad Sci U S A. 2013;110(13):4968-73.

17. Cazet A, Bobowski M, Rombouts Y, Lefebvre J, Steenackers A, Popa I, et al. The ganglioside G(D2) induces the constitutive activation of c-Met in MDA-MB-231 breast cancer cells expressing the G(D3) synthase. Glycobiology. 2012;22(6):806-16.

18. Liang YJ, Wang CY, Wang IA, Chen YW, Li LT, Lin CY, et al. Interaction of glycosphingolipids GD3 and GD2 with growth factor receptors maintains breast cancer stem cell phenotype. Oncotarget. 2017;8(29):47454-73.

19. Lefebvre T, Delannoy P. Disrupting membrane lipids composition promotes tumorigenesis: the other dark side of cholesterol and the potential implication of gangliosides. Transl Cancer Res. 2018;7(5).

20. Fitzgibbons PL, JL C. Protocol for the Examination of Biopsy Specimens From Patients With Invasive Carcinoma of the Breast. College of American Pathologists (CAP); 2019.

21. Giuliano AE, Edge SB, Hortobagyi GN. Eighth Edition of the AJCC Cancer Staging Manual: Breast Cancer. Ann Surg Oncol. 2018;25(7):1783-5.

22. Liu FS. [Histological grading and prognosis of breast cancer] Zhonghua Bing Li Xue Za Zhi. 1993;22(1):36-7.

23. Cheung IY, Cheung NK. Quantitation of marrow disease in neuroblastoma by real-time reverse transcription-PCR. Clin Cancer Res. 2001;7(6):1698-705.

24. Martinez C, Hofmann TJ, Marino R, Dominici M, Horwitz EM. Human bone marrow mesenchymal stromal cells express the neural ganglioside GD2: a novel surface marker for the identification of MSCs. Blood. 2007;109(10):4245-8.

25. Riaz-Ahmed KB. Selective Elimination of Malignant Melanoma Using the Novel Anti-tumor Agents, OSW-1 and PEITC: University of Texas; 2014.

26. Schmittgen TD, Livak KJ. Analyzing real-time PCR data by the comparative C(T) method. Nat Protoc. 2008;3(6):1101-8.

27. Laurent VE, Otero LL, Vazquez V, Camarero S, Gabri MR, Labraga $\mathrm{M}$, et al. Optimization of molecular detection of GD2 synthase mRNA in retinoblastoma. Mol Med Rep. 2010;3(2):253-9.

28. Mahata B, Banerjee A, Kundu M, Bandyopadhyay U, Biswas K. TALEN mediated targeted editing of GM2/GD2-synthase gene modulates anchorage independent growth by reducing anoikis resistance in mouse tumor cells. Sci Rep. 2015;5:9048.

29. Yeh SC, Wang PY, Lou YW, Khoo KH, Hsiao M, Hsu TL, et al. Glycolipid GD3 and GD3 synthase are key drivers for glioblastoma 
stem cells and tumorigenicity. Proc Natl Acad Sci U S A. 2016;113(20):5592-7.

30. Smith RA, Caleffi M, Albert US, Chen THH, Duffy SW, Franceschi $\mathrm{D}$, et al. Breast Cancer in Limited-Resource Countries: Early Detection and Access to Care. Breast J. 2006;12(1):16-26.

31. Kabel AM. Tumor markers of breast cancer: New prospectives. J Oncol Sci. 2017;3(1):5-11.

32. Chen K, Huang YH, Chen Jl. Understanding and targeting cancer stem cells: therapeutic implications and challenges. Acta Pharmacol Sin. 2013;34:732-40

33. Doronin, II, Vishnyakova PA, Kholodenko IV, Ponomarev ED, Ryazantsev DY, Molotkovskaya IM, et al. Ganglioside GD2 in reception and transduction of cell death signal in tumor cells. BMC Cancer. 2014;14:295.

34. Hoon DS, Kuo CT, Wen S, Wang H, Metelitsa L, Reynolds CP, et al. Ganglioside GM2/GD2 Synthetase mRNA Is a Marker for Detection of Infrequent Neuroblastoma Cells in Bone Marrow. Am J Pathol. 2001;159(2):493-500.

35. Liang YJ, Dinga Y, Levery SB, Lobaton M, Handa K, Hakomori Si. Differential expression profiles of glycosphingolipids in human breast cancer stem cells vs. cancer non-stem cells. PNAS. 2012;110(13):4968-73.

36. Kwon KM, Chung TW, Kwak CH, Choi HJ, Kim KW, Ha SH, et al. Disialyl GD2 ganglioside suppresses ICAM-1-mediated invasiveness in human breast cancer MDA-MB231 cells. Int J Biol Sci. 2017;13(3):265-75

37. Mansoori M, Roudi R, Abbasi A, Abolhasani M, Abdi Rad I, Shariftabrizi A, et al. High GD2 expression defines breast cancer cells with enhanced invasiveness. Exp Mol Pathol. 2019;109:25-35. 